\title{
Relativistic approach to neutrino-nucleus quasielastic scattering
}

\author{
Andrea Meucci, Carlotta Giusti, and Franco Davide Pacati \\ Dipartimento di Fisica Nucleare e Teorica, Università di Pavia, \\ and Istituto Nazionale di Fisica Nucleare, Sezione di Pavia, I-27100 Pavia, Italy
}

A relativistic Green's function and a distorted-wave impulse-approximation approach to charged- and neutral-current neutrino-nucleus quasielastic scattering are developed. Results for the neutrino (antineutrino) reactions on ${ }^{16} \mathrm{O}$ and ${ }^{12} \mathrm{C}$ target nuclei are presented and discussed.

PACS numbers: $25.30 . P t ; 13.15 .+\mathrm{g} ; 24.10 . \mathrm{Jv} ; 24.10 . \mathrm{Cn}$

\section{INTRODUCTION}

We are here interested in $\nu(\bar{\nu})$-nucleus scattering reactions in the quasielastic (QE) region, where the neutrino interacts with one single nucleon and the mechanisms like RPA or the excitations of the target nucleus become less important. In Sec. III a Green's function approach to describe inclusive charged-current (cc) neutrino scattering is developed [1]. In such a process, $\nu(\bar{\nu})$ 's interact with nuclei via the exchange of weak-vector bosons and charged leptons are produced in the final state. In our model the conservation of flux is preserved and final state interactions are treated consistently with an exclusive reaction. In Sec. III a relativistic distorted-wave impulse-approximation (RDWIA) calculation of semi-inclusive neutral-current (nc) $\nu$ - and $\bar{\nu}$-nucleus reactions is presented 2]. The sensitivity to the strange quark content of the nucleon weak current is discussed.

\section{THE RELATIVISTIC GREEN'S FUNCTION METHOD}

The cross section of an inclusive reaction where an incident neutrino or antineutrino interacts with a nucleus and only the outgoing lepton is detected is given by the contraction between the lepton tensor [1] and the hadron tensor, whose components are given by bilinear products of the transition matrix elements of the nuclear weak charged-current operator $J^{\mu}$. The current operator is assumed to be adequately described as the sum of single-nucleon currents, corresponding to the weak charged current

$$
j^{\mu}=\left[F_{1}^{\mathrm{V}} \gamma^{\mu}+i \frac{\kappa}{2 M} F_{2}^{\mathrm{V}} \sigma^{\mu \nu} q_{\nu}-G_{\mathrm{A}} \gamma^{\mu} \gamma^{5}+F_{\mathrm{P}} q^{\mu} \gamma^{5}\right] \tau^{ \pm},
$$

where $\tau^{ \pm}$are the isospin operators, $\kappa$ is the anomalous part of the magnetic moment, $q^{\mu}=(\omega, \boldsymbol{q})$, with $Q^{2}=|\boldsymbol{q}|^{2}-\omega^{2}$, is the four-momentum transfer, and $\sigma^{\mu \nu}=(i / 2)\left[\gamma^{\mu}, \gamma^{\nu}\right] . F_{1}^{\mathrm{V}}$ and $F_{2}^{\mathrm{V}}$ are the isovector Dirac and Pauli nucleon form factors. $G_{\mathrm{A}}$ and $F_{\mathrm{P}}$ are the axial and induced pseudoscalar form factors, which are usually parametrized as

$$
G_{\mathrm{A}}\left(Q^{2}\right)=g_{\mathrm{A}}\left(1+Q^{2} / M_{\mathrm{A}}^{2}\right)^{-2}, \quad F_{\mathrm{P}}\left(Q^{2}\right)=2 M G_{\mathrm{A}}\left(m_{\pi}^{2}+Q^{2}\right)^{-1}
$$

where $g_{\mathrm{A}}=1.267, m_{\pi}$ is the pion mass, and $M_{\mathrm{A}} \simeq(1.026 \pm 0.021) \mathrm{GeV}$ is the axial mass. Performing the contraction between the lepton and hadron tensors, the inclusive cross section for the QE $\nu(\bar{\nu})$-nucleus scattering can be written as [3]

$$
\frac{\mathrm{d} \sigma}{\mathrm{d} \varepsilon \mathrm{d} \Omega}=\frac{k \varepsilon G^{2}}{4 \pi^{2}} \cos ^{2} \vartheta_{\mathrm{c}}\left[v_{0} R_{00}+v_{z z} R_{z z}-v_{0 z} R_{0 z}+v_{T} R_{T} \pm v_{x y} R_{x y}\right],
$$


where the coefficients $v$ are obtained from the lepton tensor components. All nuclear structure information is contained in the response functions $R$, which are defined in terms of the hadron tensor components, i.e.,

$$
W^{\mu \nu}(\omega, q)=\left\langle\Psi_{0}\left|J^{\nu \dagger}(\boldsymbol{q}) \delta\left(E_{\mathrm{f}}-H\right) J^{\mu}(\boldsymbol{q})\right| \Psi_{0}\right\rangle .
$$

Introducing the Green's operator related to the nuclear Hamiltonian $H$, we have

$$
\omega^{\mu \mu}=W^{\mu \mu}(\omega, q)=-\frac{1}{\pi} \operatorname{Im}\left\langle\Psi_{0}\left|J^{\mu \dagger}(\boldsymbol{q}) G\left(E_{\mathrm{f}}\right) J^{\mu}(\boldsymbol{q})\right| \Psi_{0}\right\rangle,
$$

for $\mu=0, x, y, z$, and simliar expressions for $\omega^{0 z}$ and $\omega^{x y}[1]$.

It was shown in Refs. 1, 4] that the nuclear response in Eq. (4) can be written in terms of the single particle Green's function, $\mathcal{G}(E)$, whose self-energy is the Feshbach's optical potential. A biorthogonal expansion of the full particle-hole Green's operator in terms of the eigenfunctions of the non-Hermitian optical potential $\mathcal{V}$, and of its Hermitian conjugate $\mathcal{V}^{\dagger}$, is performed

$$
\left[\mathcal{E}-T-\mathcal{V}^{\dagger}(E)\right]\left|\chi_{\mathcal{E}}^{(-)}(E)\right\rangle=0, \quad[\mathcal{E}-T-\mathcal{V}(E)]\left|\tilde{\chi}_{\mathcal{E}}^{(-)}(E)\right\rangle=0 .
$$

Note that $E$ and $\mathcal{E}$ are not necessarily the same. The spectral representation is

$$
\mathcal{G}(E)=\int_{M}^{\infty} \mathrm{d} \mathcal{E}\left|\tilde{\chi}_{\mathcal{E}}^{(-)}(E)\right\rangle \frac{1}{E-\mathcal{E}+i \eta}\left\langle\chi_{\mathcal{E}}^{(-)}(E)\right|
$$

The hadron tensor components can be written in an expanded form in terms of the single-particle wave function, $\left|\varphi_{n}\right\rangle$, of the initial state, corresponding to the energy $\varepsilon_{n}$ and whose spectral strength is $\lambda_{n}$ as

$$
\omega^{\mu \nu}(\omega, q)=-\frac{1}{\pi} \sum_{n} \operatorname{Im}\left[\int_{M}^{\infty} \mathrm{d} \mathcal{E} \frac{1}{E_{\mathrm{f}}-\varepsilon_{n}-\mathcal{E}+i \eta} T_{n}^{\mu \nu}\left(\mathcal{E}, E_{\mathrm{f}}-\varepsilon_{n}\right)\right]
$$

where

$$
\begin{aligned}
T_{n}^{\mu \mu}(\mathcal{E}, E) & =\lambda_{n}\left\langle\varphi_{n}\left|j^{\mu \dagger}(\boldsymbol{q}) \sqrt{1-\mathcal{V}^{\prime}(E)}\right| \tilde{\chi}_{\mathcal{E}}^{(-)}(E)\right\rangle \\
& \times\left\langle\chi_{\mathcal{E}}^{(-)}(E)\left|\sqrt{1-\mathcal{V}^{\prime}(E)} j^{\mu}(\boldsymbol{q})\right| \varphi_{n}\right\rangle,
\end{aligned}
$$

for $\mu=0, x, y, z$, and simliar expressions for $\omega^{0 z}$ and $\omega^{x y}$ [1]. The factor $\sqrt{1-\mathcal{V}^{\prime}(E)}$ accounts for interference effects between different channels and allows the replacement of the mean field $\mathcal{V}$ by the phenomenological optical potential $\mathcal{V}_{\mathrm{L}}$. After calculating the limit for $\eta \rightarrow+0$, Eq. 8 reads

$$
\begin{array}{r}
\omega^{\mu \nu}(\omega, q)=\sum_{n}\left[\operatorname{Re} T_{n}^{\mu \nu}\left(E_{\mathrm{f}}-\varepsilon_{n}, E_{\mathrm{f}}-\varepsilon_{n}\right)\right. \\
\left.-\frac{1}{\pi} \mathcal{P} \int_{M}^{\infty} \mathrm{d} \mathcal{E} \frac{1}{E_{\mathrm{f}}-\varepsilon_{n}-\mathcal{E}} \operatorname{Im} T_{n}^{\mu \nu}\left(\mathcal{E}, E_{\mathrm{f}}-\varepsilon_{n}\right)\right],
\end{array}
$$

where $\mathcal{P}$ denotes the principal value of the integral.

Disregarding the square root correction, due to interference effects, The second matrix element in Eq. 9, with the inclusion of $\sqrt{\lambda_{n}}$ is the transition amplitude for the single-nucleon knockout from a nucleus in the state $\left|\Psi_{0}\right\rangle$ leaving the residual nucleus in the state $|n\rangle$. The attenuation of its strength, mathematically due to the imaginary part of the optical potential, is related to the flux lost towards the channels different from $n$. In the inclusive response this attenuation 
must be compensated by a corresponding gain, due to the flux lost, towards the channel $n$, by the other final states asymptotically originated by the channels different from $n$. This compensation is performed by the first matrix element in the right hand side of Eq. 9, where the imaginary part of the potential has the effect of increasing the strength. Similar considerations can be made, on the purely mathematical ground, for the integral of Eq. 10, where the amplitudes involved in $T_{n}^{\mu \nu}$ have no evident physical meaning when $\mathcal{E} \neq E_{\mathrm{f}}-\varepsilon_{n}$.

In an usual shell-model calculation the cross section is obtained from the sum, over all the single-particle shell-model states, of the squared absolute value of the transition matrix elements. Therefore, in such a calculation the negative imaginary part of the optical potential produces a loss of flux that is inconsistent with the inclusive process. In the Green's function approach, the flux is conserved, as the components of the hadron tensor are obtained in terms of the product of the two matrix elements in Eq. 9] the loss of flux, produced by the negative imaginary part of the optical potential in $\chi$, is compensated by the gain of flux, produced in the first matrix element by the positive imaginary part of the Hermitian conjugate optical potential in $\tilde{\chi}$. The cross sections and the response functions of the inclusive $\mathrm{QE} \nu(\bar{\nu})$-nucleus scattering are calculated from the single-particle expression of the hadron tensor in Eq. 10. After the replacement of the mean field $\mathcal{V}(E)$ by the empirical optical model potential $\mathcal{V}_{\mathrm{L}}(E)$, the matrix elements of the nuclear current operator in Eq. 9] are of the same kind as those giving the transition amplitudes of the electron induced nucleon knockout reaction [5] and the same RDWIA treatment can be used [6, 7].

As a study case, we have considered the ${ }^{16} \mathrm{O}$ target nucleus and two different values of the incident neutrino energy $E_{\nu}=500$ and $1000 \mathrm{MeV}$. In order to show up the effect of the optical potential on the inclusive reaction, the results obtained in the present approach are compared with those given by different approximations. In the simplest one the optical potential is neglected and the plane wave approximation is assumed for the final state wave functions $\chi^{(-)}$and $\tilde{\chi}^{(-)}$. In this plane wave impulse approximation (PWIA) FSI between the outgoing nucleon and the residual nucleus are completely neglected. In another approach the imaginary part of the optical potential is neglected and only the real part is included. This approximation conserves the flux, but it is inconsistent with the exclusive process, where a complex optical potential must be used. Moreover, the use of a real optical potential is unsatisfactory from a theoretical point of view, since the optical potential has to be complex owing to the presence of open channels. In Fig. 10 the differential cross sections of the ${ }^{16} \mathrm{O}\left(\nu_{\mu}, \mu^{-}\right)$reaction for $\vartheta_{\mu}=30$ degrees are displayed as a function of the muon kinetic energy $T_{\mu}$. The behavior of the calculated cross sections is similar for the different energies. The effect of the optical potential increases with $T_{\mu}$ and decreases increasing $E_{\nu}$. The result of the PWIA is about 20-30\% higher at the peak than the one of the Green's function approach. The sum of the exclusive one-nucleon emission cross sections is always much smaller than the complete result. The difference indicates the relevance of inelastic channels and is due to the loss of flux produced by the absorptive imaginary part of the optical potential. In contrast, the cross sections calculated with only the real part of the optical potential are practically the same as the ones obtained with the Green's function approach. Although the use of a complex optical potential is conceptually important from a theoretical point of view, the negligible differences given by the two results mean that the conservation of flux, that is fulfilled in both calculations, is the most important condition in the present situation. In contrast, significant differences are obtained with a real optical potential in the inclusive electron scattering [4]. The cross sections for the ${ }^{16} \mathrm{O}\left(\bar{\nu}_{\mu}, \mu^{+}\right)$ reaction are also shown for a comparison. They are always much smaller than the corresponding cross sections with an incident neutrino. 


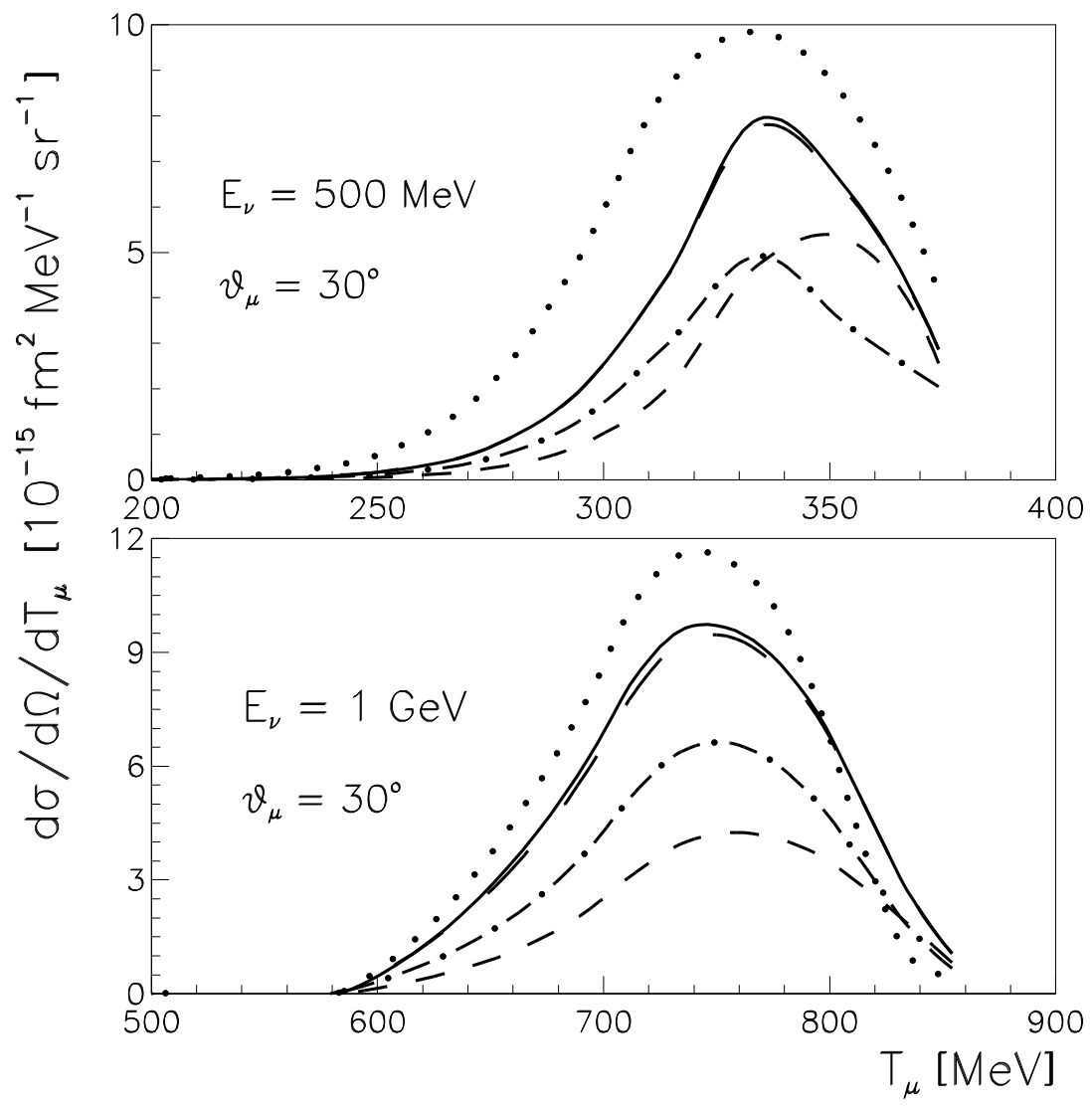

FIG. 1: The differential cross sections of the ${ }^{16} \mathrm{O}\left(\nu_{\mu}, \mu^{-}\right)$reaction for $E_{\nu}=500$ and $1000 \mathrm{MeV}$ at $\vartheta_{\mu}=$ 30 degrees. Solid lines represent the result of the Green's function approach, dotted lines give PWIA, long-dashed lines show the result with a real optical potential, and dot-dashed lines the contribution of the integrated exclusive reactions with one-nucleon emission. Short dashed lines give the cross sections of the ${ }^{16} \mathrm{O}\left(\bar{\nu}_{\mu}, \mu^{+}\right)$reaction calculated with the Green's function approach.

\section{THE NEUTRAL-CURRENT SEMI-INCLUSIVE SCATTERING}

The differential cross section for the neutral-current $\nu(\bar{\nu})$-nucleus quasielastic scattering is obtained from the contraction between the lepton and hadron tensors, as in Ref. [3]. After performing an integration over the solid angle of the final nucleon, we have

$$
\frac{\mathrm{d} \sigma}{\mathrm{d} \varepsilon \mathrm{d} \Omega \mathrm{d} \mathrm{T}_{\mathrm{N}}}=\frac{G^{2} \varepsilon^{2}}{2 \pi^{2}} \cos ^{2} \frac{\vartheta}{2}\left[v_{0} R_{00}+v_{z z} R_{z z}-v_{0 z} R_{0 z}+v_{T} R_{T} \pm v_{x y} R_{x y}\right] \frac{\left|\boldsymbol{p}_{\mathrm{N}}\right| E_{\mathrm{N}}}{(2 \pi)^{3}},
$$

where $\vartheta$ is the lepton scattering angle and $E_{\mathrm{N}}\left(\boldsymbol{p}_{\mathrm{N}}\right)$ the energy (momentum) of the outgoing nucleon. The coefficients $v$ and the responses $R$ are obtained from the lepton and hadron tensor components, respectively [2].

The transition matrix elements are calculated in the first order perturbation theory and in the impulse approximation. Thus, the transition amplitude is assumed to be adequately described as the sum of terms similar to those appearing in the electron scattering case [5, 6]. The RDWIA treatment is the same as in Refs. [6, 7]. The single-particle current operator related to the weak neutral current is

$$
j^{\mu}=F_{1}^{\mathrm{V}} \gamma^{\mu}+i \frac{\kappa}{2 M} F_{2}^{\mathrm{V}} \sigma^{\mu \nu} q_{\nu}-G_{\mathrm{A}} \gamma^{\mu} \gamma^{5}+F_{\mathrm{P}} q^{\mu} \gamma^{5}
$$


The vector form factors $F_{i}^{\mathrm{V}}$ can be expressed in terms of the corresponding electromagnetic form factors for protons $\left(F_{i}^{\mathrm{p}}\right)$ and neutrons $\left(F_{i}^{\mathrm{n}}\right)$, plus a possible isoscalar strange-quark contribution $\left(F_{i}^{\mathrm{s}}\right)$, i.e.,

$$
F_{i}^{\mathrm{V} ; p(n)}= \pm\left\{F_{i}^{p}-F_{i}^{n}\right\} / 2-2 \sin ^{2} \theta_{\mathrm{W}} F_{i}^{p(n)}-F_{i}^{s} / 2,
$$

where $+(-)$ stands for proton (neutron) knockout and $\theta_{\mathrm{W}}$ is the Weinberg angle $\left(\sin ^{2} \theta_{\mathrm{W}} \simeq 0.2313\right.$ ). The strange vector form factors are taken as [8]

$$
F_{2}^{\mathrm{s}}\left(Q^{2}\right)=\frac{F_{2}^{\mathrm{s}}(0)}{(1+\tau)\left(1+Q^{2} / M_{\mathrm{V}}^{2}\right)^{2}}, F_{1}^{\mathrm{s}}\left(Q^{2}\right)=\frac{F_{1}^{\mathrm{s}} Q^{2}}{(1+\tau)\left(1+Q^{2} / M_{\mathrm{V}}^{2}\right)^{2}},
$$

where $\tau=Q^{2} /\left(4 M_{p}^{2}\right), F_{2}^{\mathrm{s}}(0)=\mu_{\mathrm{s}}, F_{1}^{\mathrm{s}}=-\left\langle r_{\mathrm{s}}^{2}\right\rangle / 6$, and $M_{\mathrm{V}}=0.843 \mathrm{GeV}$. The quantity $\mu_{\mathrm{s}}$ is the strange magnetic moment and $\left\langle r_{\mathrm{s}}^{2}\right\rangle$ the squared "strange radius" of the nucleon. The axial form factor is expressed as

$$
G_{\mathrm{A}}\left(Q^{2}\right)=\frac{ \pm g_{\mathrm{A}}-g_{\mathrm{A}}^{s}}{2\left(1+Q^{2} / M_{\mathrm{A}}^{2}\right)^{2}},
$$

where $g_{\mathrm{A}}^{\mathrm{s}}$ describes possible strange-quark contributions.

In order to separate the effects of the strange-quark contribution and of FSI on the cross sections, it was suggested in Refs. 9] to measure the ratio of proton to neutron yields, as this ratio is expected to be less sensitive to distortion effects than the cross sections themselves. Moreover, from the experimental point of view the ratio is less sensitive to the uncertainties in the determination of the incident neutrino flux. In Fig. 2 the ratio for an incident neutrino is displayed as a function of the outgoing nucleon kinetic energy both in RDWIA and RPWIA. The ratio is very sensitive to $g_{\mathrm{A}}^{\mathrm{s}}$ and exhibits a maximum at $T_{N} \simeq 0.6 E_{\nu}$, which, however, corresponds to values where the cross sections are small. The effect is sensibly reduced for $g_{\mathrm{A}}^{\mathrm{s}}=-0.10$ with respect to $g_{\mathrm{A}}^{\mathrm{s}}=-0.19$. An enhancement of the ratio of $\simeq 15 \%$ is produced by FSI. This result is due both to Coulomb distortion and to the different coupling of the optical potential with proton and neutron currents. It means that the argument of looking for the strange-quark content in this ratio is strengthened by distortion, but the possibility to fix the exact value of the contribution is affected by the uncertainties due to FSI.

Finally, in Fig. 3 we compare our results with the data of the BNL 734 experiment [10]. Experimental results were presented in the form of a flux-averaged differential cross section per momentum transfer squared $Q^{2}$. Our results are shown with $g_{\mathrm{A}}^{\mathrm{s}}=-0.19$ and without the strangequark contribution. We give also the effect of including the strange vector form factors, $F_{1}^{\mathrm{s}}$ and $F_{2}^{\mathrm{s}}$, with $F_{1}^{\mathrm{s}}=-\left\langle r_{\mathrm{s}}^{2}\right\rangle / 6=0.53 \mathrm{GeV}^{-2}, F_{2}^{\mathrm{s}}(0)=-0.40$ [1]], and the $Q^{2}$ dependence given in Eq. 14. The strange-quark contribution produces an enhancement of the cross sections, which makes them slightly higher than the experimental data. The strange weak magnetic contribution decreases the cross section, while the axial and weak electric components give an enhancement.

[1] A. Meucci, C. Giusti, and F.D. Pacati, Nucl. Phys. A 739, 277 (2004).

[2] A. Meucci, C. Giusti, and F.D. Pacati, Nucl. Phys. A 744, 307 (2004).

[3] J.D. Walecka, in Muon Physics, Vol. II, edited by V.H. Hughes and C.S. Wu (Academic Press, New York, 1975), p. 113.

[4] A. Meucci, F. Capuzzi, C. Giusti, and F.D. Pacati, Phys. Rev. C 67, 054601 (2003).

[5] S. Boffi, et al., Electromagnetic Response of Atomic Nuclei, Oxford Studies in Nuclear Physics, Vol. 20 (Clarendon, Oxford, 1996); S. Boffi, et al., Phys. Rep. 226, 1 (1993). 


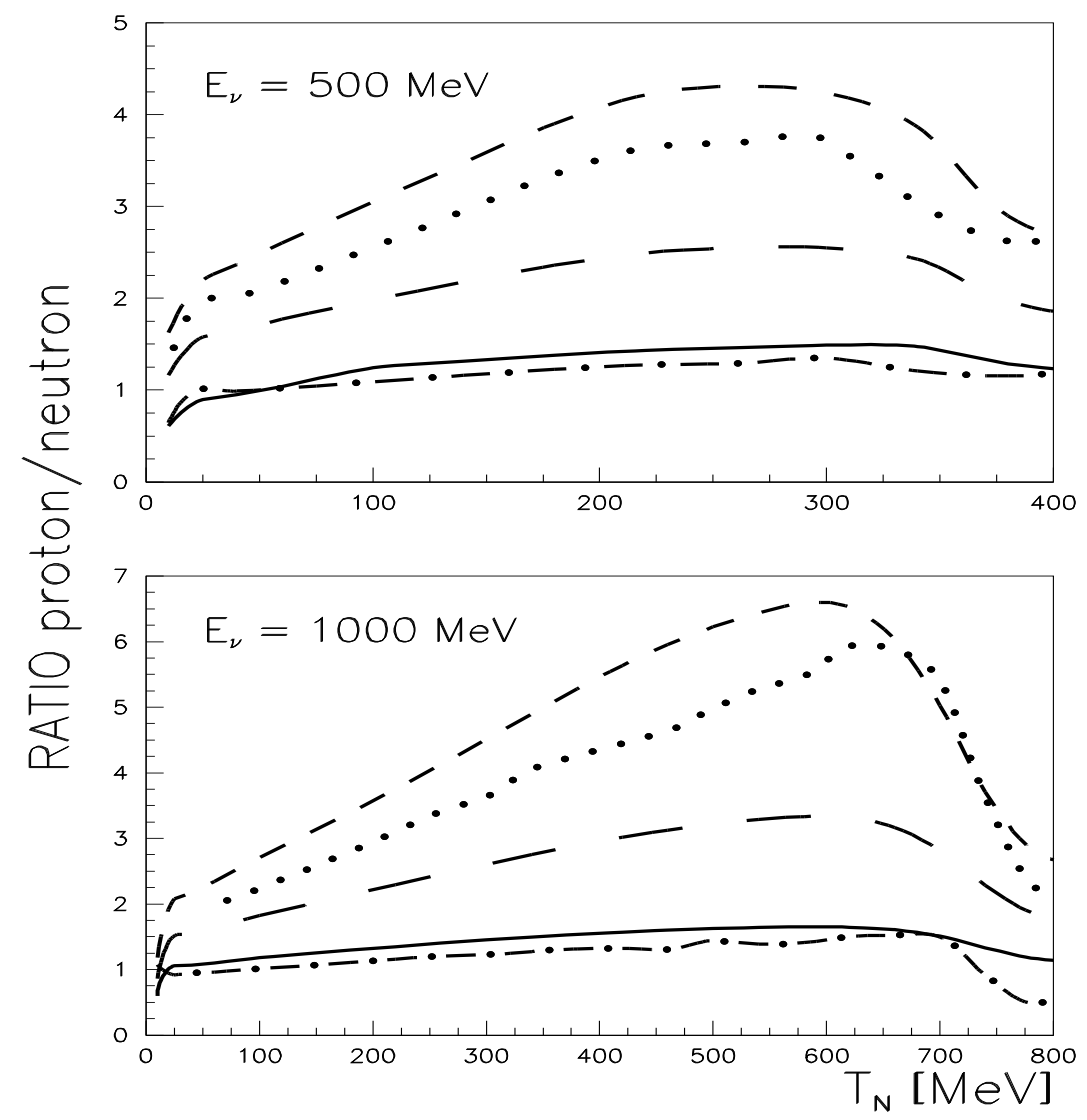

FIG. 2: Ratio of proton to neutron total cross sections of the $\nu(\bar{\nu})$ quasielastic scattering on ${ }^{12} \mathrm{C}$ as a function of the incident neutrino (antineutrino) energy. Solid and dashed lines are the results in RDWIA with $g_{\mathrm{A}}^{\mathrm{s}}=0$ and $g_{\mathrm{A}}^{\mathrm{s}}=-0.19$. Dot-dashed and dotted lines are the same results but for an incident antineutrino. Longdashed line corresponds to neutrino scattering with $g_{\mathrm{A}}^{\mathrm{s}}=-0.10$.

[6] A. Meucci, C. Giusti, and F.D. Pacati, Phys. Rev. C 64, 014604 (2001).

[7] A. Meucci, C. Giusti, and F.D. Pacati, Phys. Rev. C 64, 064615 (2001).

[8] G.T. Garvey, et al., Phys. Rev. C 48, 1919 (1993).

[9] G.T. Garvey, et al., Phys. Lett. B 289, (1992) 249; C.J. Horowitz, et al., Phys. Rev. C 48, 3078 (1993); W.M. Alberico, et al., Nucl. Phys. A 623, 471 (1997); W.M. Alberico, et al., Phys. Lett. B 438, 9 (1998).

[10] L.A. Ahrens, et al., Phys. Rev. D 35, 785 (1987).

[11] G.T. Garvey, et al., Phys. Rev. C 48, 761 (1993). 


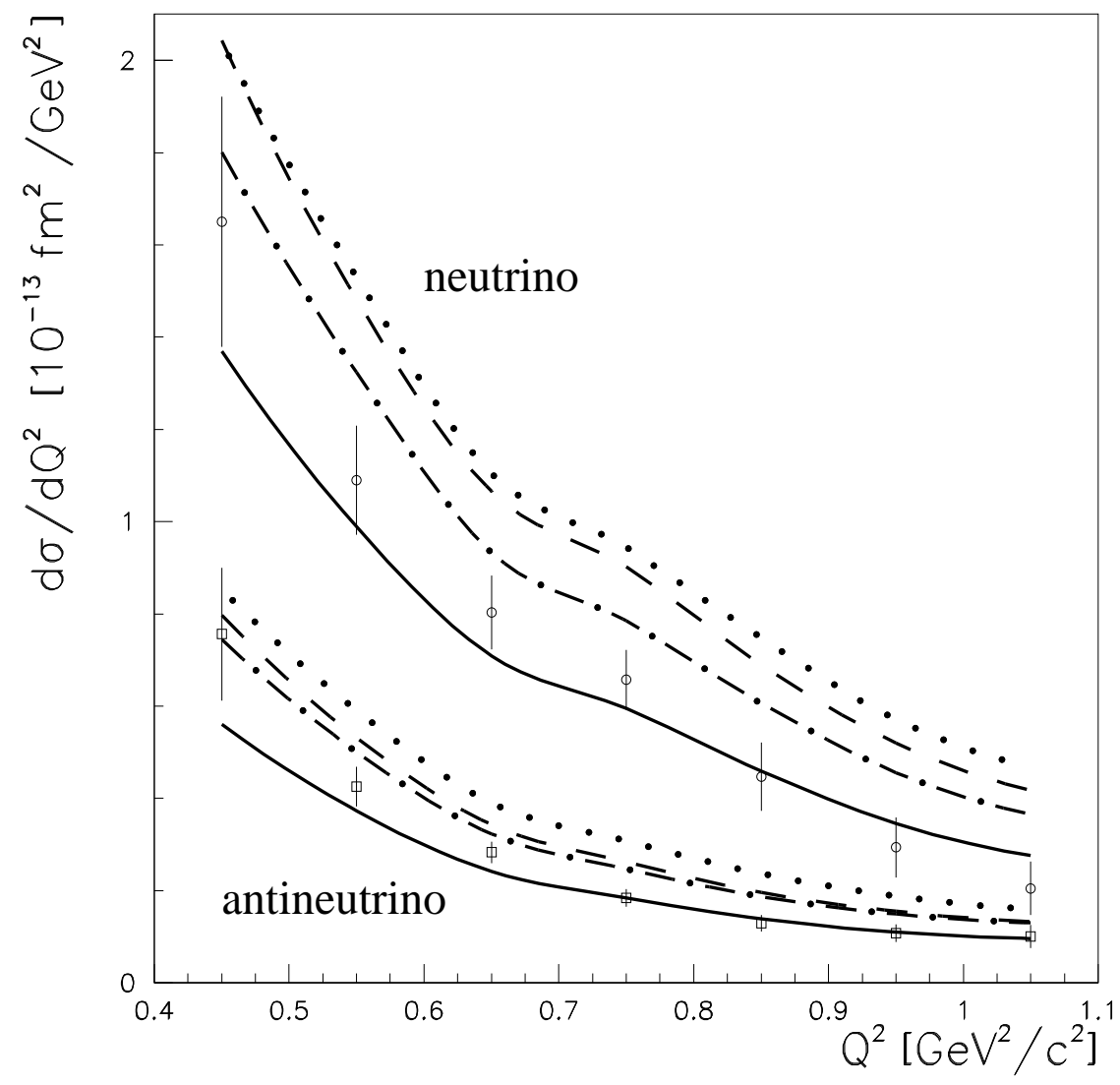

FIG. 3: Differential cross sections of the $\nu(\bar{\nu})$ quasielastic scattering, flux-averaged over BNL spectrum 10], as a function of the momentum transfer squared. The four upper curves are for incident neutrino and the four lower ones for incident antineutrino. Solid lines are the results with no strangeness contribution, dashed lines with $g_{\mathrm{A}}^{\mathrm{s}}=-0.19$, dot-dashed lines with $g_{\mathrm{A}}^{\mathrm{s}}=-0.19$ and $F_{2}^{\mathrm{s}}(0)=-0.40$, dotted lines with $g_{\mathrm{A}}^{\mathrm{s}}=-0.19$, $F_{2}^{\mathrm{s}}(0)=-0.40$ and $F_{1}^{\mathrm{s}}=-\left\langle r_{\mathrm{s}}^{2}\right\rangle / 6=0.53 \mathrm{GeV}^{-2}$. Experimental data from Ref. [10]. The errors correspond to statistical and $Q^{2}$-dependent systematic errors added in quadrature and do not include $Q^{2}$-independent systematic errors. 